\title{
DESARROLLO Y APLICACIÓN DE XEROGELES DE CARBONO EN EL TRATAMIENTO DE EFLUENTES ACUOSOS.
}

\author{
Alondra Fabiola Mar Ortiz ${ }^{a}$, Jacob Josafat Salazar Rábago ${ }^{a^{*}}$, Margarita Loredo Cancino ${ }^{a}$, Manuel \\ Sánchez Polo ${ }^{b}$, Felipe de Jesús Cerino Córdova ${ }^{c}$
}

${ }^{a}$ Universidad Autónoma de Nuevo León, Facultad de Ciencias, Av. Universidad s/n Cd. Universitaria, 66455 San Nicolás de los Garza, Nuevo León, México

${ }^{b}$ Universidad de Granada, Facultad de Ciencias, Departamento de Química Inorgánica, 18071 Granada, España.

${ }^{c}$ Universidad Autónoma de Nuevo León, Facultad de Ingeniería Mecánica y Eléctrica, Av. Universidad s/n Cd. Universitaria, 66455 San Nicolás de los Garza, Nuevo León, México

e-mail: jacob.sakazarrb@uanl.edu.mx

\section{Resumen}

Los xerogeles de carbono son materiales con una fácil síntesis, a los cuales se les puede realizar diversas modificaciones en sus propiedades texturales y ópticas. Así mismo estos materiales han tenido un amplio uso en diversas áreas de la ciencia como son la catálisis, procesos de adsorción, fotocatálisis y electroquímica. El presente trabajo, es una breve revisión al uso de estos materiales en los tratamientos de aguas residuales debido a su excelente comportamiento en la remoción de contaminantes.

Palabras clave: Xerogeles de carbono, síntesis, adsorción, procesos avanzados de oxidación.

\section{Abstract}

Carbon xerogels are materials with an easy synthesis, to which various modifications can be made to their textural and optical properties. Likewise, these materials have been widely used in various areas of science such as catalysis, adsorption processes, photocatalysis and electrochemistry; The use of these materials in wastewater treatment processes will be addressed due to their excellent behavior in the removal of contaminants.

Keywords: Carbon xerogels, synthesis, adsorption, advanced oxidation processes.

\section{Introducción}

La introducción de materiales funcionales en la industria de la transformación y servicios ha ido en aumento en los últimos años. Concretamente, aquellos producidos a base de carbono han presentado un aumento significativo en su uso en diversas áreas de la ciencia, tales como catálisis, fotocatálisis, electroquímica, ingeniera de los materiales, etc. El carbono es un elemento tetravalente no metálico, el cual existe abundantemente en la corteza terrestre y la atmósfera, lo que lo convierte en un elemento accesible para sus aplicaciones en múltiples procesos a nivel industrial y de investigación.

En la Figura 1 se puede observar la evolución del número de publicaciones anuales con materiales de carbono en revisas indexadas en SCOPUS. Como se puede observar en esta figura, existe un incremento sostenido en las investigaciones relacionadas con el diseño, síntesis, evaluación y aplicación de dichos materiales.
Los materiales sintetizados a base de carbono pueden clasificarse de acuerdo a sus aplicaciones las cuales son muy variadas debido a las diversas propiedades físicas y químicas de las que se les puede dotar, por mencionar algunos, se tiene su empleo como adsorbentes [1, 2], soportes catalíticos [3], catalizadores $[4,5]$, fotocatalizadores $[6,7]$, entre otras aplicaciones.

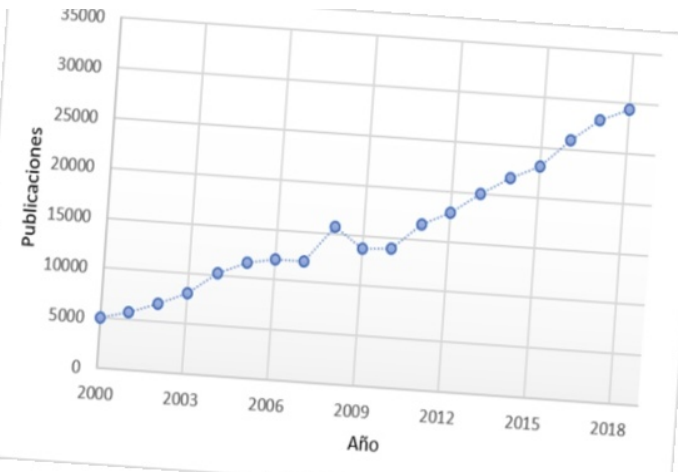

Figura 1. Gráfico de publicaciones realizadas para materiales a base de carbono. Fuente: Scopus. 
Además, los materiales carbonosos pueden clasificarse según su origen, así por ejemplo un gran número de carbones activados son obtenidos a partir de biomasas lignocelulósicas [8,9], o a partir de fibras como el nylon [10]. Los xerogeles orgánicos de carbono (XOC) son obtenidos a través de un proceso de policondensación de compuestos aromáticos hidroxilados y aldehídos, seguido de un curado a presión atmosférica y condiciones térmicas moderadas, el cual se puede carbonizar en función de la aplicación que se le desee dar y con ello se obtiene el xerogel de carbono (XC). Dada la sencillez de la ruta de síntesis y la alta pureza del material resultante se han investigado diversas aplicaciones de estos materiales (Figura 2). En dicha figura se puede observar que la mayor parte de ellas se relacionan con el área del conocimiento ingenieril, especialmente con los tópicos de la ciencia de los materiales y la química.

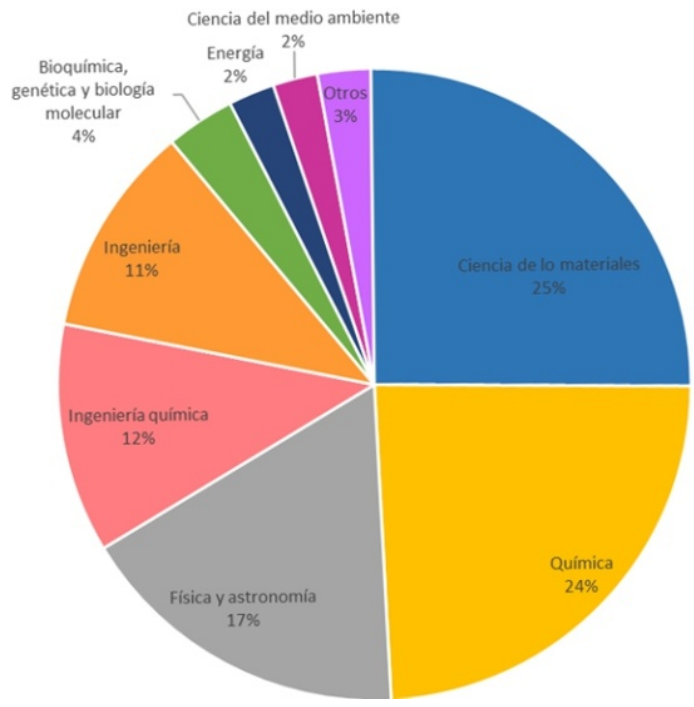

Figura 2. Áreas de investigación y aplicación de los xerogeles de carbono. Fuente: Scopus.

Como ya se mencionó en el párrafo anterior, las áreas predominantes son la ciencia de los materiales y de la química, las cuales se han basado más en el estudio de ciertas propiedades que se les puede proporcionar a los materiales, así como la modificación y evaluación de las condiciones de síntesis para generar una eficiencia en el tiempo de preparación y utilización de reactivos. Es por eso que en el caso del tratamiento de aguas residuales, numerosas investigaciones se han centrado en la aplicación de xerogeles de carbono a procesos de adsorción para la remoción de contaminantes (colorantes, metales pesados, contaminantes emergentes, entre otros) [1, 11-13].
En algunos casos, la aplicación de procesos de adsorción no logra eliminar por completo el problema del contaminante por lo que se recomienda, en el caso de contaminantes orgánicos, la aplicación de tecnologías que logren mineralizar al compuesto tóxico $[14,15]$, en este sentido cobran relevancia los procesos avanzados de oxidación (PAOs), los cuales promueven la degradación del contaminante orgánico a través de la acción de especies de alto poder oxidante como son los radicales hidroxilos.

Dentro de los PAOs sobresalen aquellos procesos fotoquímicos que emplean la radiación solar como fuente activante del catalizador debido a que este método busca la descomposición de las moléculas contaminantes o la transformación de los compuestos a otros menos dañinos, además de implementar un enfoque más sostenible y sustentable basado en los doce principios de la química verde. Un gran número de materiales se han desarrollado buscando optimizar la generación de especies oxidantes en la región visible del espectro electromagnético, sobresaliendo los xerogeles orgánicos e inorgánicos de carbono, los cuales han presentado bajos requerimientos energéticos para lograr la promoción de especies oxido-reductoras, así como susceptibilidad a la regeneración y altas tasas de remoción [5, 16-18].

Considerando lo anterior, en el presente trabajo se expone un análisis del desarrollo y aplicación de los xerogeles de carbono, en la última década, en procesos de tratamientos de aguas, con un especial enfoque en procesos fotoquímicos. En primera instancia se abordará la naturaleza de dichos materiales y cómo en función de las condiciones de síntesis varían sus propiedades texturales, para después analizar sus aplicaciones.

\section{Naturaleza de los xerogeles}

Tal como se ilustra en la Figura 3, los geles orgánicos de carbono son preparados a partir de la policondensación de compuestos aromáticos hidroxilados (fenol, catecol, resorcinol o hidroquinona) y aldehídos (formaldehído o furfural) en un disolvente, generalmente agua, en condiciones alcalinas o neutras, seguido del proceso de curado y secado. De acuerdo a las condiciones en que se realice el curado, al material resultante se le denomina xerogel, criogel o aerogel, si se realizó bajo condiciones subcríticas, criogénicas o supercríticas, respectivamente [19]. El material resultante es un gel orgánico de carbono, los cuales pueden grafitizarse a temperaturas mayores a los $300^{\circ} \mathrm{C}$ convirtiéndose en geles de carbono. Dicho proceso se lleva a cabo, por 
lo general, a través de una pirólisis que conduce al desarrollo de una estructura microporosa que resulta en elevadas superficies específicas. Por regla general los xerogeles de carbono (XC) presentan mejores propiedades texturales que los xerogeles orgánicos (XOC) [20].

Los XC además de sus propiedades texturales, también poseen propiedades mecánicas y químicas deseables en diversas áreas de investigación. De entre algunas de las diversas propiedades que podemos destacar, resaltan su elevada área específica $\mathrm{y}$ volumen de poros, alta conductividad eléctrica, baja densidad y las diversas formas de obtención como monolitos, polvo, microesferas, películas delgadas, etcétera [14].

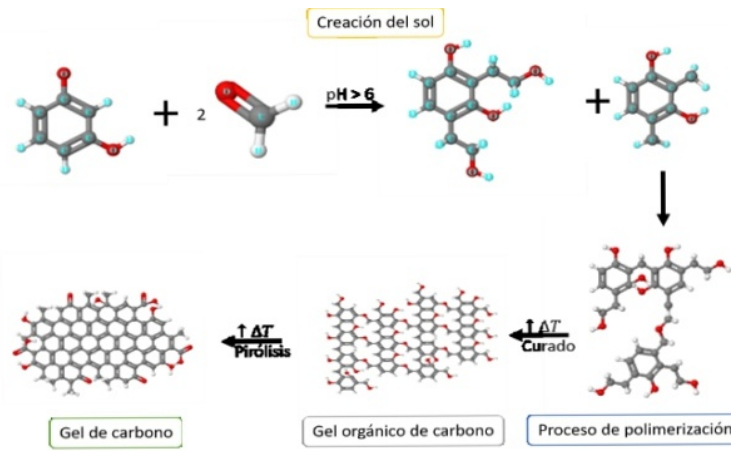

Figura 3. Esquematización del proceso de síntesis de los geles de carbono.

Las propiedades texturales de los XC se pueden modificar dependiendo los factores intrínsecos y extrínsecos. Dentro de los factores intrínsecos se consideran los precursores utilizados, su naturaleza y complejidad, el tipo y concentración de grupos funcionales; por otro lado las condiciones de operación como la concentración de precursores, $\mathrm{pH}$ de la solución, condiciones de gelificación y curado, el proceso de secado y carbonización/activación del material son considerados factores extrínsecos [21, 22].

Por otra parte, la incorporación de diversos agentes dopantes a la matriz polimérica tales como óxidos o iones metálicos, así como diversos compuestos orgánicos ha sido reportada por diversos autores [2327], encontrando que este dopaje favorece la modificación de las características superficiales del material, principalmente sus propiedades ópticas.

En la Tabla 1 se presentan las relaciones molares, catalizadores y temperatura de pirólisis empleadas en la síntesis de diversos $\mathrm{XC}$, así mismo se indica el área específica reportada mediante la cual se pueden realizar diversas conclusiones respecto a la temperatura de carbonización aplicada y los factores anteriormente mencionados. Orellana García et al. [20] realizaron la síntesis de XOC y XC, empelando resorcinol, formaldehído, agua y acetatos de $\mathrm{Fe}, \mathrm{Co}$, $\mathrm{Ni}$ como catalizadores, para posteriormente realizar la carbonización a $900{ }^{\circ} \mathrm{C}$ con un flujo de nitrógeno de $150 \mathrm{~cm}^{3}$ por 5 horas, concluyendo que la carbonización promueve la aparición de microporos y aumenta el área específica, sin embargo la actividad fotocatalítica se ve disminuida al carbonizar dichos materiales.

De igual modo, reporta Carrasco Díaz et al. [28] una serie de catalizadores con hierro mediante diversas condiciones de síntesis, siendo el método de impregnación el que favoreció que los XC obtuvieran una mayor área específica y cantidad de mesoporos y microporos. Por otra parte, Hongqi Sun et al. [29] estudiaron mediante dos técnicas la inserción de cobalto, en xerogeles preparados a partir de resorcinol, furfural, hexametilentetramina y ácido acético siendo utilizados estos últimos dos como catalizadores. La primera de ellas consistió en la inserción del ion $\mathrm{Co}$ en el gel orgánico húmedo obteniendo así un XC dopado; asimismo, la segunda vía consistió en la impregnación del $\mathrm{XC}$ con $\mathrm{Co}$ presente en solución acuosa. Los resultados revelaron que el material impregnado mostraba un área específica de $2408 \mathrm{~m}^{2} / \mathrm{g}$ la cual fue 5 veces superior al XC dopado; esto se correlaciono con el incremento en la cantidad de microporos y una constante de degradación menor.

De igual manera, Bailón García et al. [30] trabajaron con diferentes cantidades de $\mathrm{TiO}_{2}$ en $\mathrm{XC}$ para la degradación del colorante naranja $\mathrm{G}$, obteniendo que los compósitos presentaban de manera uniforme la dispersión de nanopartículas de $\mathrm{TiO}_{2}$ fase anatasa en el soporte de carbono, siendo los XC con 30 y $40 \%$ en peso total los que presentaron mejores propiedades ópticas al poderse activar en la luz visible, además de una mayor área específica.Es así por lo que se concluye, que el método de síntesis puede brindar distintas propiedades en los materiales carbonosos que en el siguiente punto se podrán conocer algunas de sus aplicaciones actuales.

\section{Aplicaciones de los xerogeles de carbono}

Los xerogeles de carbono como ya se ha venido mencionando tienen varias aplicaciones. A continuación, se muestra una breve descripción de los tratamientos de aguas donde se han utilizado estos 
materiales, así como un condensado de los diversos trabajos reportados para cada sección.

\section{Procesos de adsorción}

En los últimos años las industrias han crecido y al mismo tiempo se ha visto aumentada la contaminación presente en las aguas, debido a las descargas de diversos compuestos, creando un nuevo desafío para las industrias tratadoras de aguas. Además, la demanda de agua de alta calidad ha aumentado significativamente. Como resultado, se han desarrollado nuevas tecnologías [35] dentro de las que podemos mencionar:

Los procesos de adsorción basados en la eliminación completa en la mayoría de los casos del contaminante mediante un adsorbente que es donde el adsorbato interacciona con la interfase superficial del mismo. En el caso de los xerogeles de carbono se ha demostrado que presentan un sistema desordenado y no uniforme, con formación de mesoporos en la etapa de síntesis, mientras que los microporos por lo general son resultado del proceso de carbonización [1].

En la Tabla 2 se presentan los valores de la capacidad máxima de adsorción de algunos xerogeles de algunos contaminantes orgánicos, la cual nos ayuda a conocer la capacidad que tiene el material para adsorber y de esta manera remover los diferentes contaminantes orgánicos. Algunos contaminantes como los fármacos se encuentran presentes en aguas residuales, siendo de suma importancia la remoción de este tipo de sustancias del agua, debido a que aun a bajas concentraciones puede llegar a causar diversas alteraciones tanto a la biota marina como a los seres terrestres.

Es por eso que la adsorción de diclofenaco se evaluó con un XC tratado con ácido sulfúrico el cual presentó una capacidad máxima de adsorción de 80 $\mathrm{mg} / \mathrm{g}$ en donde predominaron las interacciones electrónicas, en el mismo artículo publicado por Álvarez et al. [11] también se realizó la adsorción de cafeína que es un alcaloide del grupo de las xantinas, el cual presentó valores máximos de adsorción de $182.5 \mathrm{mg} / \mathrm{g}$ atribuyéndose al xerogel de carbono tratado con urea el cual le da la propiedad de ser un material con mayor afinidad por los compuestos orgánicos debido a la presencia de bases de Lewis en su superficie. El estudio concluye que ambos materiales pueden llegar a tener una muy buena competitividad comparado con carbones activados comerciales al presentar buenas tasas de remoción.

Aparte de ello, Ptaszkowska-Koniarz et al. [1] estudió la adsorción del colorante rodamina $\mathrm{B}$, utilizado principalmente por su fluorescencia en la industria, con xerogeles modificados mediante grupos amino y cloruro de cobre (II). Los resultados mostraron que la muestra con la combinación de ambos dopantes fue la que presentó la mejor adsorción, al igual que el que contiene etilendiamina presentando una $\mathrm{Q}_{\mathrm{e}}$ de 132 $\mathrm{mg} / \mathrm{g}$. La adición de la sal del metal y la presencia de grupos amino en su superficie aumenta la capacidad de sorción del material.

Tabla 1. Relaciones de síntesis de XC, a base de Resorcinol (R), Formaldehido (F) y agua (W) como reactivos de síntesis .

\begin{tabular}{|c|c|c|c|c|}
\hline Relación & Catalizador $(\mathbf{C})$ & Carbonización $\left({ }^{\circ} \mathbf{C}\right)$ & $\mathbf{S}_{\mathbf{B E T}}\left(\mathbf{m}^{2} / \mathbf{g}\right)$ & Ref. \\
\hline $\mathrm{R} / \mathrm{F}=0.5$ & & & & \\
$\mathrm{R} / \mathrm{S}=20$ & $\mathrm{Co}\left(\mathrm{NO}_{3}\right)_{2}$ & 800 & 403 & {$[31]$} \\
& & & & \\
\hline $\mathrm{R} / \mathrm{F}=0.5$ & $\mathrm{Ac}(\mathrm{Fe})_{1}$ & & $338_{1}$ & \\
$\mathrm{R} / \mathrm{W}=0.07$ & $\mathrm{Ac}(\mathrm{Ni})_{2}$ & & $382_{2}$ & {$[20]$} \\
$1 \%$ en peso total del \\
catalizador
\end{tabular}




\begin{tabular}{|c|c|c|c|c|}
\hline $\begin{array}{c}\mathrm{R} / \mathrm{F}=0.5 \\
\mathrm{R} / \mathrm{C}=300 \\
\mathrm{R} / \mathrm{W}=0.07\end{array}$ & $\begin{array}{l}\mathrm{Li}_{2} \mathrm{CO}_{3} \\
\mathrm{Cs}_{2} \mathrm{CO}_{3}\end{array}$ & & $\begin{array}{l}569 \\
616\end{array}$ & [33] \\
\hline $\begin{array}{c}\mathrm{R} / \mathrm{F}=0.5 \\
\mathrm{R} / \mathrm{W}=0.07 \\
\mathrm{R} / \mathrm{S}=4.5\end{array}$ & $\begin{array}{l}\text { Isopropóxido de titanio (IV) como } \\
\text { precursor de } \mathrm{TiO}_{2} \text { y Spam } 80(\mathrm{~S})\end{array}$ & & $\begin{array}{c}\text { CTi10 } \\
523 \\
\text { CTi20 } \\
480 \\
\text { CTi30 } \\
481 \\
\text { CTi30s2h } \\
401\end{array}$ & [30] \\
\hline $\begin{array}{c}\mathrm{R} / \mathrm{F}=0.5 \\
\mathrm{R} / \mathrm{C}=1000 \\
\mathrm{R} / \mathrm{S}=125\end{array}$ & $\begin{array}{l}\mathrm{Na}_{2} \mathrm{CO}_{3} \text { y } \mathrm{N} \text {-cetil-N, } \mathrm{N}, \mathrm{N}- \\
\text { trimetilamonio bromuro como } \\
\text { precursor }(\mathrm{S})\end{array}$ & 1000 & 468 & [34] \\
\hline
\end{tabular}

Procesos Avanzados de Oxidación

Los sistemas de tratamiento de aguas residuales aplicados normalmente, solo se basan en procesos físicos y bioquímicos para la eliminación de diversos contaminantes del medio acuoso. Sin embargo diversos estudios han demostrado que estos procedimientos no son eficaces para la remoción total de contaminantes orgánicos e inorgánicos[36]. Es por ello que es necesario incluir un procedimiento terciario que logre la eliminación de los contaminantes, esto es en el caso de los compuestos orgánicos su mineralización. Por otra parte, los procesos avanzados de oxidación (PAOs) han sido definidos por Glaze et al. [37] como aquellos procesos que involucran la generación de radicales hidroxilos en cantidad suficiente para la mineralización de los contaminantes objetivo, es por ello que numerosas investigaciones se han realizado en esta área.
Como ya se mencionó con anterioridad, este tipo de tratamientos se han utilizado para eliminar compuestos orgánicos de diversas fuentes, como son: aguas subterráneas, aguas residuales municipales e industriales [35]. Muchas veces los PAOs presentan la desventaja de ser un método costoso por los reactivos o materiales ocupados. Es por eso que la utilización de la oxidación húmeda catalítica con peróxido de hidrógeno es uno de los procesos más económicos y efectivos utilizados para la remoción de diversos contaminantes de las aguas residuales, mediante el uso de $\mathrm{H}_{2} \mathrm{O}_{2}$ y un catalizador metálico, $\left(\mathrm{Fe}^{2+}, \mathrm{Fe}^{3+}\right.$, etc.) para la generación de radicales hidroxilo o perhidroxilo ( $\mathrm{OH} \cdot \mathrm{OOH} \cdot$ ) [38]. Sin embargo, el uso de catalizadores en fase homogénea conlleva un proceso posterior de remoción de estos, por lo que el proceso heterogéneo cobra una mayor relevancia en su aplicabilidad.

Tabla 2. Valores reportados de la capacidad de adsorción (Qe) de diversos XC.

\begin{tabular}{|c|c|c|}
\hline Contaminante & $\left(\mathbf{Q}_{\mathbf{e}} \mathbf{)} \mathbf{~ m g} / \mathbf{g}\right.$ & Ref. \\
\hline Cafeína & 182.5 & {$[11]$} \\
\hline Rodamina B & $91-132$ & {$[1]$} \\
\hline Ciprofloxacina & 112 & {$[2]$} \\
\hline Diclofenaco & 80 & {$[11]$} \\
\hline
\end{tabular}

Ribeiroa et al. [15] estudiaron la conjugación entre la oxidación húmeda del peróxido de hidrógeno y el empleo de XC dopados con iones de Fe, Co y una mezcla de ambos, encontrando que la inserción de metales en la matriz carbonosa de los XC favorece la degradación de los contaminantes, reportando para el 
material con un dopaje bimetálico $\mathrm{Fe} / \mathrm{Co}$ una tasa de degradación del $98.5 \%$ para una solución de 4nitrofenol en un lapso de 30 minutos, comparado con los monometálicos que presentaron tasas de degradación menores[24]. Asimismo Fathy et al. [25] llevaron a cabo la impregnación de níquel en los xerogeles para la degradación de naranja de metilo, la cual se ve directamente relacionada con la dosis de $\mathrm{H}_{2} \mathrm{O}_{2}$, catalizador, temperatura y concentración inicial del colorante, pudiéndose tener un porcentaje de degradación mayor al $90 \%$ al paso de 30 minutos a un $\mathrm{pH}$ ácido y tener hasta cuatro ciclos de reúso del material.

Por otra parte, la presencia de grupos funcionales de nitrógeno en la superficie del XC es extremadamente importante para las aplicaciones catalíticas de materiales de carbono. Debido a que los electrones del $\mathrm{N}$ superficial interaccionan con el oxígeno adsorbido, propiciando a la formación de radicales altamente reactivos en presencia de agua, es por eso que Rocha et al. [5] trabajaron en la degradación de ácido oxálico con XC dopados con nitrógeno, los cuales presentaron una alta eficiencia para el de oxidación húmeda, logrando una completa remoción del contaminante en menos de 45 minutos, demostrando de dicha manera que la presencia de una fuente de nitrógeno contribuye positivamente a la formación de radicales y aumenta el rendimiento catalítico del proceso, siendo más notorio en el de oxidación húmeda.

En la Tabla 3 se presentan diferentes xerogeles dopados para la degradación de colorantes, medicamentos de uso habitual y compuestos químicos, así como su porcentaje de degradación al utilizar el método de oxidación húmeda catalítica. Los porcentajes de degradación reportados para la mayoría de los xerogeles ocupados se encuentran entre el 70 y $100 \%$, dando a concluir que este tipo de proceso de oxidación y el uso de este tipo de materiales ha favorecido de manera positiva a la remoción de contaminantes orgánicos del medio acuoso.

La fotodegradación de compuestos orgánicos es considerado un PAO el cual puede llevarse a cabo en forma homogénea o empleando catalizadores en fase heterogénea, ha este proceso habitualmente se le conoce como fotocatálisis. Tal como se ilustra en la Figura 4 el proceso fotocatalítico se basa en la generación de especies oxidantes a partir de la

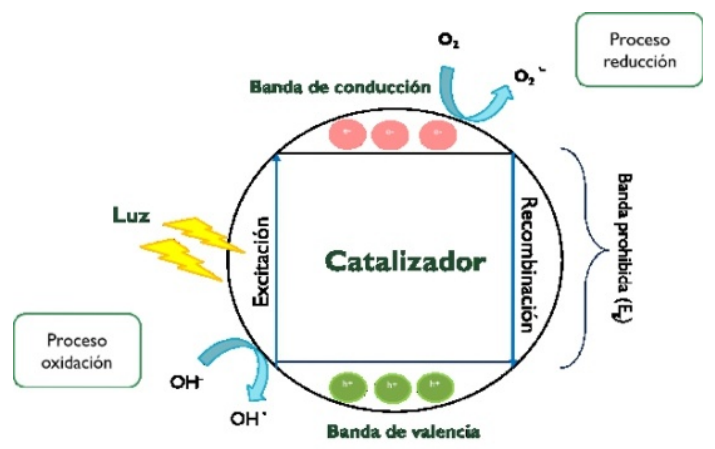

Figura 4. Proceso de activación de un fotocatalizador para la formación del par electrón-hueco.

activación del catalizador, para que esto ocurra el material debe ser irradiado con una determinada longitud de onda para la activación del mismo, dicha longitud de onda es conocida como banda prohibida y señala la barrera que limita el paso de electrones de la banda de valencia a la de conducción [35].

Tabla 3. XC ocupados para procesos de oxidación húmeda catalítica con $\mathrm{H}_{2} \mathrm{O}_{2}$.

\begin{tabular}{|c|c|c|c|}
\hline Contaminante & Dopante & \% Deg. & Ref. \\
\hline Naranja de metilo & $\mathrm{Ni}$ & $<90 \%$ & {$[26]$} \\
\hline Verde de metilo & Ce- Nanobarras & $100 \%$ & {$[25]$} \\
\hline Paracetamol & $\mathrm{Fe}$ & $85-90 \%$ & {$[28]$} \\
\hline Fenol & $\mathrm{Co}$ & $100 \%$ & {$[29]$} \\
\hline
\end{tabular}

Investigaciones recientes recomiendan que en este tipo de materiales la denominada banda prohibida sea lo más pequeña posible para favorecer la activación del fotocatalizador en la región visible del espectro electromagnético; con ello se lograría contribuir a disminuir el impacto económico de estos procesos, ya que se podría emplear como fuente de irradiación al sol en comparación del uso de luz artificial que representa una mayor inversión energética y por ende monetaria.[39]. En la Tabla 4 se muestra de manera resumida, los dopantes, la energía de banda prohibida $\mathrm{y}$ tasas de remoción de diversos $\mathrm{XC}$ utilizados para la degradación completa o parcial de diversos contaminantes como herbicidas, colorantes y fármacos. Los agentes dopantes son de naturaleza metálica, sobresaliendo el $\mathrm{TiO}_{2}, \mathrm{ZnO}, \mathrm{Fe}_{2} \mathrm{O}_{3}, \mathrm{Ni}$, y $\mathrm{CuO}$ los cuales han mostrado altas tasas de 
degradación para los diversos contaminantes $[16,30$, 40-42].

Bailón García et al. [30] utilizaron xerogeles de carbono- $\mathrm{TiO}_{2}$ para la fotodegradación de naranja $\mathrm{G}$, empleando una lámpara de luz visible donde se obtuvo una degradación del $79 \%$ y $90 \%$ para los xerogeles con una relación de 30 y $40 \%$ en masa de $\mathrm{TiO}_{2}$ respectivamente. Utilizando de igual manera el $\mathrm{TiO}_{2}$ y $\mathrm{TiO}_{2}$-porfirina como dopantes para xerogeles se reportaron valores del 10 y $40 \%$ de degradación respectivamente para el p-nitrofenol utilizando irradiación ultravioleta-visible, concluyendo que los xerogeles con $\mathrm{TiO}_{2}$-porfirina presentan una mayor eficiencia fotocatalítica cuatro veces mejor que los que simplemente están sintetizados con dióxido de titanio[17].

Así mismo el uso de metales como el níquel en los $\mathrm{XC}$ ha favorecido a la degradación de contaminantes, Tabla 4. Porcentajes de degradación en diferentes contaminantes con XC.

\begin{tabular}{|c|c|c|c|c|c|}
\hline Contaminante & Dopante & Banda prohibida (eV) & Radiación & \% Deg. & Ref. \\
\hline Amitrol & $\mathrm{Ni}$ & 3.66 & Ultravioleta & $90 \%$ & {$[20]$} \\
\hline Naranja G & $\mathrm{TiO}_{2}$ & 2.8 & Visible & $79 \%$ & {$[30]$} \\
\hline Azul de metileno & $\mathrm{ZnO}$ & 3.22 & Solar & 96 y $60 \%$ & {$[41]$} \\
\hline 2,4-D Sal sódica & $\mathrm{CuO}-\mathrm{TiO}_{2}$ & 3.23 & UV/Vis & $97-100 \%$ & {$[40]$} \\
\hline Diuron & $\mathrm{Ni}$ & 3.66 & Solar & $95 \%$ & {$[42]$} \\
\hline Azul de metileno & $\mathrm{Nb}_{2} \mathrm{O}_{5}$ & $3.14-3.39$ & Visible & $65-70 \%$ & {$[43]$} \\
\hline
\end{tabular}

El óxido de niobio, así como otros óxidos de metales presentan un alto gap de energía, haciendo que sean reactivos interesantes; al ayudar a la generación de electrones-hueco en el fotocatalizador con una menor energía de activación, por consiguiente, la degradación de azul de metileno fue realizada con compósitos de $\mathrm{Nb}_{2} \mathrm{O}_{5}$ con los cuales se obtuvo que presentaban una energía de banda prohibida muy similar a la que presenta el $\mathrm{TiO}_{2}$, concluyendo que estos novedosos materiales reportados por Perciani de Moraes et al. [43] son fotoactivos tanto en la región ultravioleta como en la visible.

\section{Conclusiones}

Los materiales carbonosos se han estado trabajando por más de dos décadas en las diversas áreas de las ciencias ingenieriles, químicas y de materiales debido a su gran versatilidad en aplicaciones y fácil acceso a las fuentes de materia prima. Dentro de los materiales desarrollados sobresalen los xerogeles de carbono (XC), los cuales presentan la peculiaridad de ser materiales con una ruta de síntesis sencilla y a los cuales se les puede dotar de las características como lo reportó López Ramon et al. [6] al realizar la fotodegradacion de amitrol, herbicida ocupado para el control de la maleza en los campos agrícolas. Para el proceso de fotodegradación se tomaron en cuenta el porcentaje de adsorción, fotólisis y la sinergia al emplear el xerogel, obteniendo un porcentaje de degradación del $96 \%$ con un $\mathrm{pH}$ básico y una concentración de amitrol de $0.30 \mathrm{mmol} / \mathrm{L}$. En un trabajo posterior descrito para la degradación del herbicida Diuron mediante radiación solar se obtuvieron porcentajes de fotodegradación de alrededor del $88.5 \%$ en comparación con la fotólisis aplicada al herbicida (78.6\%), pudiendo concluir que al utilizar el xerogel con níquel se incrementó la tasa catalítica producida incrementando los sitios activos en la superficie para la degradación del contaminante [42]. texturales (tamaño de poro, área específica, etc.) y/o fisicoquímicas (tipo de sitios activos, energía de activación, luminiscencia, etc.) en función de la aplicación que se les desee dar.

En el área de tratamiento de aguas residuales, los XC han sido ocupados principalmente en los procesos de adsorción y de oxidación avanzada. En en este último caso destacan sus aplicaciones en la oxidación húmeda catalítica con peróxido de hidrógeno y la fotocatálisis. En el caso de los procesos fotocatalíticos, la revisión reveló que es necesario dopar o impregnar al material carbonoso con óxidos o iones metálicos, logrando así disminuir su energía de banda prohibida y promoviendo su activación en la región visible del espectro electromagnético. Con ello se ha logrado la degradación de diversos contaminantes bajo radiación solar, lo que abre una ventana de posibilidades en la aplicación y desarrollo de estos materiales en la industria de la purificación de efluentes acuosos.

\section{Referencias}

1. Ptaszkowska-Koniarz, M., Goscianska, J. \& Pietrzak, R. Colloids Surf., A: Physicochemical and Engineering Aspects (2018), 543, 109-117.

2. Carabineiro, S. A. C., Thavorn-amornsri, T., 
Pereira, M. F. R., Serp, P. \& Figueiredo, J. L. Catal. Today (2012), 186, 29-34.

3. Mobini, S., Meshkani, F. \& Rezaei, M. Chem. Eng. Sci. (2019), 197, 37-51.

4. Kumari, M. \& Saroha, A. K. J. Environ. Chem. Eng. (2019), 7 (3), 103121

5. Rocha, R.P., Restivo, J., Sousa J.P.S.órfão, M. F R. Pereira, \& J. L. Figueiredo. Catal. Today (2015), 241, 73-79.

6. Álvarez, M. A., Orellana-García, F., LópezRamón, M. V., Rivera-Utrilla, J. \& Sánchez-Polo, M. Arabian J. Chem. (2018), 11, 564-572.

7. Acosta-Rangel, A., Sánchez-Polo, M., Polo, A. M. S., Rivera-Utrilla, J. \& Berber-Mendoza, M. S. Chem. Eng. J. (2018), 344, 21-33.

8. Sun, B.,Yuan, Y., Li, H.,Li, X.,Zhang, C.,Guo F.,Liu, X.,Wang, K.,Zhao, X.S. Chem. Eng. J. (2019), 371, 55-63.

9. Wang, X. C. Jiang, B. Hou, Y. Wang, C. Hao, and J. Wu. Chemosphere (2018), 206, 587-596.

10. Racho, P. \& Phalathip, P. Energy Procedia (2017), 118, 195-200.

11. Álvarez, S., Ribeiro, R. S., Gomes, H. T., Sotelo, J. L. \& García, J. Chemical Engineering Research and Design (2015), 95, 229-238.

12. Zhou, G., Tian, H., Sun, H., Wang, S. \& Buckley, C. E. Chem. Eng. J. (2011), 171, 1399-1405.

13. Wong, S., Ngadi, N., Inuwa, I. M. \& Hassan, O. J. Cleaner Prod. (2018), 175, 361-375.

14. Orge, C. A., órfão, J. J. M. \& Pereira, M. F. R. A Appl. Catal., B. (2012), 126, 22-28.

15. Ribeiro, Rui S.,Frontistis,Z.,Mantzavinos, D., Venieri, Danae,A., Maria,K., Ioannis,S., Adrián M.T.,Faria, J. L.,Gomes, H., T. Appl. Catal., B. (2016), 199, 170-186.

16. Carrasco-Díaz, M. R., Castillejos-López, E., Cerpa-Naranjo, A. \& Rojas-Cervantes, M. L. Microporous Mesoporous Mater. (2017), 237, 282-293.

17. Tasseroul, L., Pirard, S., Lambert, S., Páez, C., Poelman, D. Pirard, J.-P.\& Heinrichs, B. Chem. Eng. J. (2012), 191,441-450

18. Huang, G., Li, W. \& Song, Y. J Sol-Gel Sci Technol (2018), 86: 175-186.

19. Job, N., Théry, A., Pirard, R., Marien, J., Kocon, L., Jean-Noël,R., François,B., Jean-Paul, P. Carbon (2005), 43, 2481-2494.

20. Orellana-García, F. Álvarez,M. A., LópezRamón,M. V., Rivera-Utrilla J., Sánchez-Polo M., \& M. Á. Fontecha-Cámara Appl. Catal., B. (2016), 181, 94-102.

Figueiredo, J. L. \& Pereira, M. F. R.. J. Energy Chem. (2013), 22, 195-201.
Brinker, C. J. \& Scherer, G. W. Sol-gel science: the physics and chemistry of sol-gel processing. (Academic Press, 1990).

23. Salazar-Rábago, J. J. Sánchez-Polo, M., RiveraUtrilla, J., Leyva-Ramos, R., Ocampo-Pérez, R., \& Carrasco-Marin, F. Chem. Eng. J. (2016), 306, 289-297.

Ribeiro, R. S., Silva, A. M. T., Figueiredo, J. L., Faria, J. L. \& Gomes, H. T. Catal. Today (2017), 296, 66-75.

25. Fathy, N. A., El-Khouly, S. M., Hassan, N. A. \& Awad, R. M. S. J. Water Process Eng. (2017), 16, 21-27.

Rashwan, W. E., Fathy, N. A. \& Elkhouly, S. M. J. Taiwan Inst. Chem. Eng. (2018), 88, 234-242.

27. Lu,C., Huang,Y. H., Hong, J. S., Wu, Y. J., Li, J., \& Cheng, J. P. J. Colloid Interface Sci. (2018), 524, 209-218.

28. Carrasco-Díaz, M. R., Castillejos-López, E., Cerpa-Naranjo, A. \& Rojas-Cervantes, M. L. Microporous Mesoporous Mater. (2017), 237, 282-293.

29. Sun, H., Tian, H., Hardjono, Y., Buckley, C. E. \& Wang, S. Catal. Today (2012), 186, 63-68.

30. Bailón-García, E. Elmouwahidi, A., Álvarez, M. A., Carrasco-Marín, F., Pérez-Cadenas, A. F., \& Maldonado-Hódar, F. J. Appl. Catal., B. (2017), 201, 29-40.

31. Jin, H., Li, J., Chen, F., Gao, L., Zhang, H., Liu, D., Liu, Q. Electrochim. Acta (2016), 222, 438445.

32. Pérez-Cadenas, A. F., Ros, C. H., Morales-Torres, S., Pérez-Cadenas, M., Kooyman, P. J., MorenoCastilla, C., \& Kapteijn, F. Carbon (2013), 56, 324-331.

33. Morales-Torres, S., Maldonado-Hódar, F. J., Pérez-Cadenas, A. F. \& Carrasco-Marín, F. $M$ Microporous Mesoporous Mater. (2012), 153, 24 29.

34. Osińska, M., Krawczyk, P., Rozmanowski, T. \& Gurzęda, B. Appl. Surf. Sci. (2019), 481, 545-553.

35. Ray M.B., Chen J.P., Wang L.K., \& Pehkonen S.O. (2006) Advanced Oxidation Processes. In: Wang L.K., Hung YT., Shammas N.K. (eds) Advanced Physicochemical Treatment Processes. Handbook of Environmental Engineering, vol 4. Humana Press

36. Delgado, N., Navarro, A., Capparelli, A., Peñuela G. \& Marino, D. TECNOLOGÍAS ALTERNATIVAS PARA LA REMOCIÓN DE CONTAMINANTES EMERGENTES EN AGUAS RESIDUALES

37. Glaze, W. H., Kang, J.-W. \& Chapin, D. H. Ozone Sci. Eng. (1987), 9, 335-352.

38. A. Quintanilla, C.M. Domínguez, J.A. Zazo, J. A Casas, J. J. R. Bol. Grupo Español Carbón 
(2014),31, 6 .

39. Herrmann, J. M. J. Photochem. Photobiol., A (2010), 216, 85-93.

40. López-Ayala, S., Rincón, M. E., Quiroz Alfaro, M. A., Bandala, E. R., Méndez Rojas, M. A., \& Castaño, V. J. Photochem. Photobiol., A (2015), 311, 166-175.

41. de Moraes, N. P., Bacetto, L., dos Santos, G., Pinto da Silva, M., Machado J., Campos, T. M. B.,Thim, G. P. R., Liana A. Ceram. Int. (2019), 45, 3657-3667.

42. López-Ramón, M. V., Rivera-Utrilla, J., SánchezPolo, M., Polo, A.M.S., Mota, Antonio J., Orellana-García, F., Álvarez, M.A.Sci. Total Environ. (2019), 650, 1207-1215.

43. de Moraes, N. P., da Silva, M. L. C. P. \& Rodrigues, L. A. Mater. Lett. (2018), 228, 486489. 